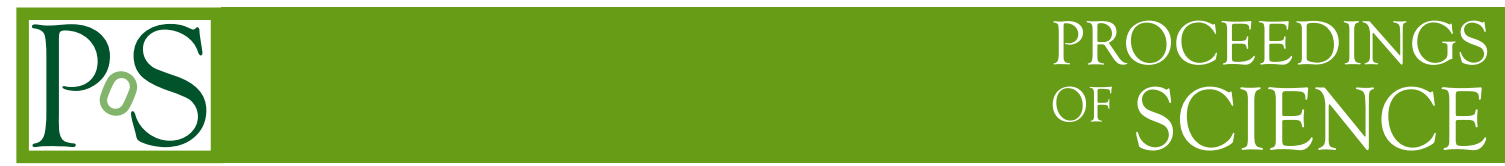

\title{
Luminosity measurement with the ATLAS experiment at the LHC
}

Joseph Carter*, on behalf of the ATLAS Collaboration

Department of Physics, University of Toronto,

60 St. George St, Toronto, Ontario, M5S 1A7, Canada

E-mail: joey.carter@cern.ch

Many physics measurements made by the ATLAS experiment at the LHC require a precise measurement of the integrated luminosity of the data sample. Here we describe the ATLAS luminosity measurement for the full Run-2 dataset, including the absolute calibration of the luminosity scale using the van der Meer scan technique in dedicated LHC running, the extrapolation of this calibration to high-luminosity data-taking, and studies of the long-term stability.

40th International Conference on High Energy physics - ICHEP2020

July 28 - August 6, 2020

Prague, Czech Republic (virtual meeting)

${ }^{*}$ Speaker 


\section{Introduction}

A precise measurement of the integrated luminosity is a key component of the ATLAS [1] physics programme at the LHC. A precise offline luminosity measurement is important for all physics analyses, particularly for precision cross-section measurements where it is often one of the leading sources of uncertainty. Accurate online luminosity monitoring is essential as well, both for the operation of the LHC (e.g. for performance optimization and levelling), and for the experiment (e.g. for optimization of the trigger configuration).

The ATLAS luminosity measurement is based on an absolute calibration of the primary luminosity-sensitive detectors in low-luminosity runs with specially-tailored LHC conditions using the van der Meer (vdM) method [2,3]. A calibration transfer procedure is then used to transport this calibration to the physics data-taking regime at high luminosity. The vdM calibration was performed once per year during Run-2 data-taking, and relative comparisons of the luminosities measured by different detectors were used to set limits on any possible change of the calibration through the year. Finally, the integrated luminosity and uncertainty for the whole Run-2 data-taking period was derived, taking into account correlations between the uncertainties in each of the component years.

This luminosity calibration procedure is performed for each of the Run-2 datasets sharing common running conditions, such as centre-of-mass energy and pileup profile. This note describes the preliminary luminosity calibration for the $\sqrt{s}=13 \mathrm{TeV}$ high-pileup $p p$ dataset [4], the $\sqrt{s}=$ $13 \mathrm{TeV}$ low-pileup $p p$ dataset (recorded for precision $W$ boson measurements, where high pileup degrades the detector resolution in $W \rightarrow \ell v$ decays), and the $\sqrt{s}=5.02 \mathrm{TeV}$ low-pileup $p p$ dataset (which served as $p p$ reference data with the same nucleon-nucleon collision energy as the Run-2 $\mathrm{PbPb}$ dataset for the LHC heavy-ion programme) [5].

\section{Luminosity detectors and algorithms}

The luminosity calibration relies on multiple redundant luminosity detectors and algorithms, which have complementary capabilities and different systematic uncertainties. The LUCID Cherenkov detector [6] provided the primary bunch-by-bunch luminosity measurement during Run 2. This was complemented by bunch-by-bunch measurements from the ATLAS beam conditions monitor (BCM) diamond detectors, and from offline measurements of the multiplicity of reconstructed charged particles in randomly-selected bunch-crossings (track counting). The ATLAS calorimeters provided bunch-integrated measurements based on quantities proportional to instantaneous luminosity: liquid-argon (LAr) gap currents in the electromagnetic endcap (EMEC) and forward (FCal) calorimeters, and photomultiplier currents from the scintillating-tile hadronic calorimeter (TILE).

The LUCID detector is composed of 16 photomultiplier tubes (PMTs) in each forward arm of the ATLAS detector (side A and side C), placed at approximately $z= \pm 17 \mathrm{~m}$ from the interaction point. ${ }^{1}$ Cherenkov light is produced in the quartz windows of the PMTs, which are coated with

${ }^{1}$ ATLAS uses a right-handed coordinate system with its origin at the nominal interaction point in the centre of the detector. The $x$ axis direction points towards the centre of the LHC ring, the $y$ axis vertically upwards, and the $z$ axis lies along the beam line. Pseudorapidity is defined in terms of the polar angle $\theta$ as $\eta=-\ln \tan \theta / 2$, and transverse momentum and energy are defined relative to the beamline as $p_{\mathrm{T}}=p \sin \theta$ and $E_{\mathrm{T}}=E \sin \theta$. 
${ }^{207} \mathrm{Bi}$ radioactive sources that provide a calibration signal. The LUCID detector was read out with dedicated electronics providing luminosity counts for each of the 3564 nominal LHC bunch slots. These counts were integrated over "luminosity blocks," which have a typical length of 60 seconds. Several luminosity "algorithms" were used to convert the raw signals from the PMTs to a luminosity measurement, combining the information from several PMTs in various ways. For example, the BiHitOR algorithm was used in 2016 and 2017, counting the average number of hits per bunch crossing summed over four Bi-calibrated PMTs on the A-side and four more on the C-side. In 2018, a significant number of PMTs stopped working during the course of the data-taking year, and a single PMT on the C-side (C12) was used for the baseline luminosity estimate. In all cases, the raw hit counts were converted into a visible interaction rate per bunch crossing $\mu_{\mathrm{vis}}$, which is related to the per-bunch instantaneous luminosity $\mathcal{L}_{b}$ via

$$
\mathcal{L}_{b}=\frac{\mu_{\mathrm{vis}} f_{r}}{\sigma_{\mathrm{vis}}},
$$

where $f_{r}$ is the LHC revolution frequency (11246 Hz for protons), and $\sigma_{\text {vis }}$ is a calibration constant, which represents the absolute luminosity calibration of the given algorithm, and is determined experimentally via the vdM calibration discussed in Section 3. The total instantaneous luminosity is the sum of $\mathcal{L}_{b}$ over all colliding bunch pairs.

\section{Absolute luminosity calibration}

The absolute luminosity calibration of LUCID, corresponding to the determination of the visible cross-section $\sigma_{\text {vis }}$ for each of the LUCID algorithms, was derived using dedicated vdM scan sessions during special LHC fills in each data-taking year. The instantaneous luminosity for a single colliding bunch pair, $\mathcal{L}_{b}$, is given in terms of $\mathrm{LHC}$ beam parameters by

$$
\mathcal{L}_{b}=\frac{f_{r} n_{1} n_{2}}{2 \pi \Sigma_{x} \Sigma_{y}}
$$

where $n_{1}$ and $n_{2}$ are the numbers of protons in the beam- 1 and beam- 2 colliding bunches, and $\Sigma_{x}$ and $\Sigma_{y}$ are the convolved beam sizes in the horizontal and vertical planes [3]. The quantity $\Sigma_{x}$ can be determined using the instantaneous luminosity $R(\Delta x)$ measured as a function of the separation $\Delta x$ between the two beams in the horizontal plane:

$$
\Sigma_{x}=\frac{1}{\sqrt{2 \pi}} \frac{\int R(\Delta x) \mathrm{d} \Delta x}{R(0)},
$$

and similarly for $\Sigma_{y}$ with a scan in the vertical plane. Since the normalisation of $R(\Delta x)$ cancels out in Eq. (3), any quantity proportional to luminosity can be used to determine the scan curve. The calibration of a given algorithm (i.e. its $\sigma_{\text {vis }}$ value) can then be determined by combining Eqs. (1) and (2) to give

$$
\sigma_{\mathrm{vis}}=\mu_{\mathrm{vis}}^{\max } \frac{2 \pi \Sigma_{x} \Sigma_{y}}{n_{1} n_{2}}
$$

where $\mu_{\mathrm{vis}}^{\max }$ is the visible interaction rate per bunch crossing at the peak of the scan curve. A single pair of $x-y$ vdM scans thus suffices to measure $\sigma_{\text {vis }}$ for each algorithm active during the scan. 


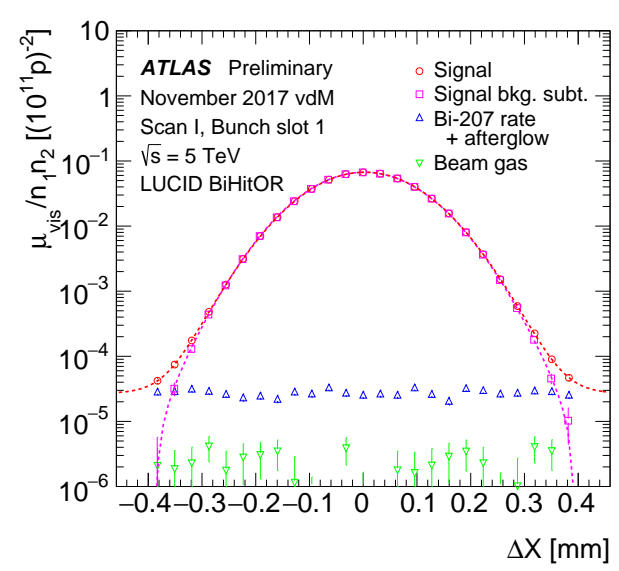

(a)

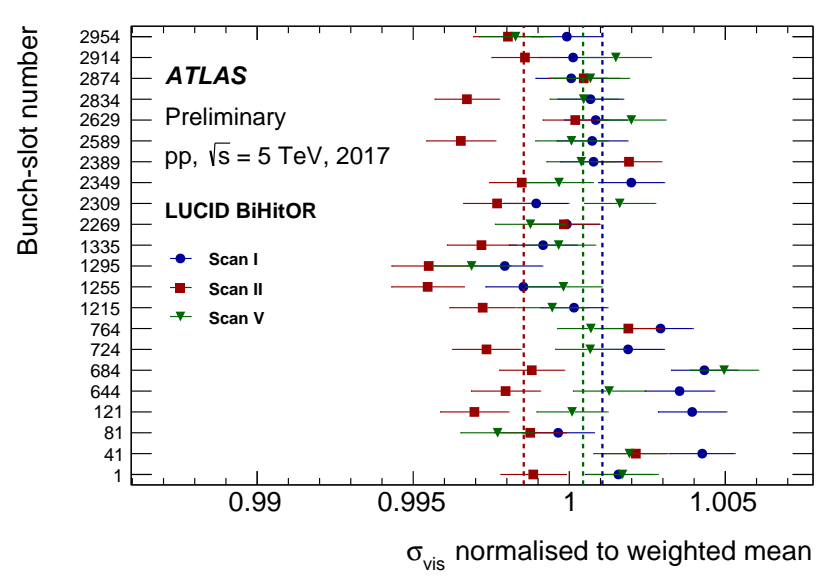

(b)

Figure 1: Example of a typical ATLAS vdM session from 2017 at $\sqrt{s}=5 \mathrm{TeV}$. (a) Visible interaction rate $\mu_{\text {vis }}$ per unit bunch population product $n_{1} n_{2} v s$. horizontal beam separation $\Delta X$, as measured by the LUCID BiHitOR algorithm. (b) Ratios of bunch-by-bunch visible cross-sections to the weighted mean of $\sigma_{\text {vis }}$ for all colliding bunch pairs and on-axis scans [5].

Figure 1a shows a typical scan profile from 2017 at $\sqrt{s}=5 \mathrm{TeV}$ from a single colliding bunch pair during a scan in the $x$-direction. The curve shows a fit (using a Gaussian $\times$ polynomial function) to the background-subtracted visible interaction rate $\mu_{\mathrm{vis}}$, normalized by the bunch population product $n_{1} n_{2}$. The latter was measured using the LHC DC current transformers (DCCT) to obtain the total current in each beam and the fast beam-current transformers (FBCT) to resolve the total current into the fractions in individual bunches. Figure $1 \mathrm{~b}$ shows the consistency of the $\sigma_{\mathrm{vis}}$ values measured for each colliding bunch pair in all three of the on-axis vdM scans from this session. The bunch-by-bunch and scan-to-scan inconsistencies are taken as systematic uncertainties.

A number of corrections are required in the vdM calibration procedure to account for deviations from ideal conditions, with each of these methods contributing its own systematic uncertainties. One example is the non-factorization correction. The vdM formalism described by Eqs. (2-4) assumes that the particle densities in each bunch can be factorized into independent horizontal and vertical components, which is not always the case. A correction is determined from combined fits to both the luminosity and the position, orientation and shape of the luminous region (characterized by the spatial distribution of primary vertices) at each scan step in both on- and off-axis vdM scans. The correction factors to $\sigma_{\text {vis }}$ are typically $<1 \%$, with uncertainties of $0.2-0.5 \%$. Other leading sources of systematic uncertainties in the $\mathrm{vdM}$ method include, but are not limited to, the choice of scan curve fit model, beam-beam effects, the length-scale calibration, beam-position jitter, orbit-drift corrections and the consistency between luminosity algorithms and detectors.

\section{Calibration transfer to physics data-taking conditions}

The procedures discussed above provide the absolute calibration of the LUCID luminosity measurements for low- $\mu$ data-taking with a limited number of isolated bunches. However, the LUCID detector suffers from significant non-linearity, and requires a correction of $O(10 \%)$ in the physics data-taking regime. This effect can clearly be seen by studying the ratios between 
instantaneous luminosities measured with LUCID and with other detectors, and how they change as a function of instantaneous luminosity or $\mu$.

The effects of this non-linearity were corrected by comparing LUCID to track-counting luminosity measurements, which are known from comparisons with EMEC and TILE to have much smaller non-linearity as a function of $\mu$. A single long high-luminosity physics fill, near in time to the vdM calibration period in each year of data-taking, was used to derive a correction of the form

$$
\mu_{\text {corr }}=p_{0} \mu_{\text {uncorr }}+p_{1} \mu_{\text {uncorr }}^{2},
$$

where $\mu_{\text {uncorr }}$ is the uncorrected and $\mu_{\text {corr }}$ the corrected LUCID $\mu$ value, and the parameters $p_{0}$ and $p_{1}$ were obtained from a linear fit to the ratio of $\mu$ values measured by track-counting and LUCID, $R=\mu_{\text {track }} / \mu_{\text {uncorr }}$, as a function of $\mu_{\text {uncorr }}$. The track-counting luminosity was first normalized to the absolute luminosity measured by LUCID in parts of the vdM fill with stable, almost-constant luminosity, where the beams were colliding head-on, thus ensuring that $R=1$ at low luminosity with isolated bunches. The 2018 correction to the $\mathrm{C} 12$ algorithm is shown in Figure 2a. This calibration transfer procedure implicitly assumes that track-counting suffers from no significant non-linearity between the low-luminosity vdM and high-luminosity physics regimes. This assumption was verified by comparing the ratio of the track-counting luminosity to that from the TILE E cells in the vdM fill and a closely-following physics fill. An example of this procedure for the $\sqrt{s}=13 \mathrm{TeV}$ high- $\mu$ dataset from 2017 is illustrated in Figure 2b. In this case, a relative difference of 1.3\% was observed, which was interpreted as an upper limit on the non-linearity of the track-counting luminosity measurement and thus assigned as a systematic uncertainty on the correction applied to LUCID at high luminosity. A calibration transfer was still required for the low- $\mu$ datasets, mainly due to bunch-train and LHC crossing-angle effects, although the size of the correction was smaller-typically only $\sim 1 \%$.

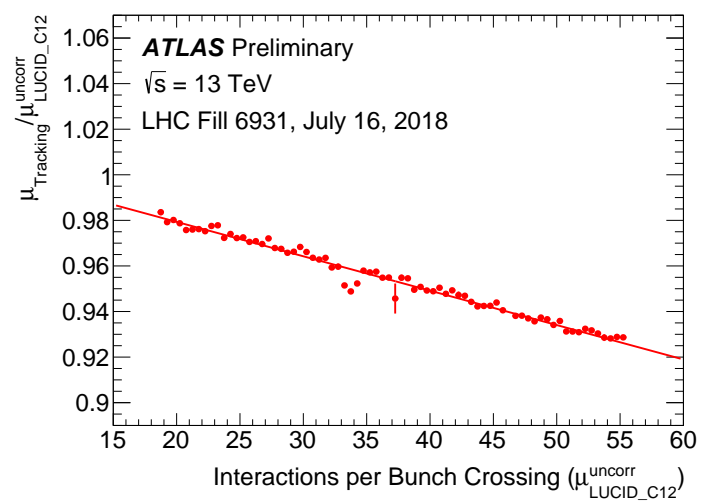

(a)

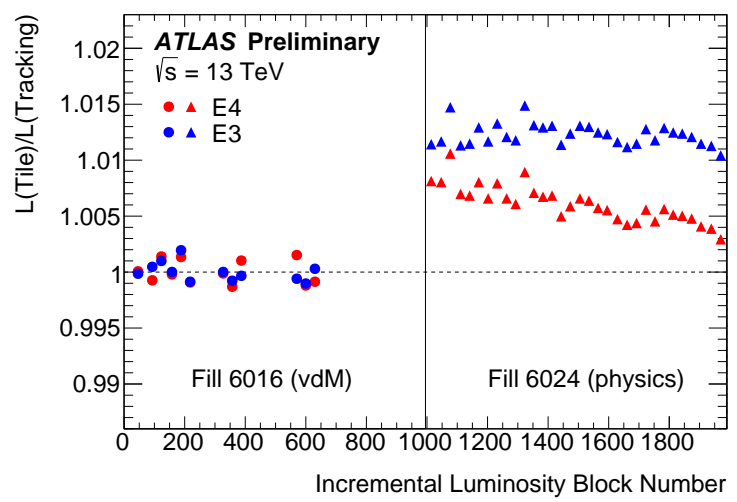

(b)

Figure 2: The calibration transfer: (a) Ratio of track-counting to LUCID C12 luminosity in LHC physics fill 6931. (b) Ratios of the instantaneous luminosity measured by TILE E-cell scintillators to that from track-counting, in a vdM fill (LHC fill 6016) and a closely-following physics fill (LHC fill 6024) [4].

\section{Long-term stability}

The vdM calibration, followed by the calibration transfer to the physics regime, determines the absolute LUCID luminosity scale at one point during the data-taking year. To quantify any possible 
drifts over the year, the LUCID integrated luminosity estimates were compared for each physics run with those from other subdetectors, after renormalizing the other luminosity estimates to agree with LUCID in a long "reference" run close to the vdM scan. A conservative "stability band" uncertainty is then assigned such that it encloses the bulk of the differences between LUCID and any of the other luminosity measurements. This stability uncertainty is illustrated as the yellow band in Figure 3 for (a) the $\sqrt{s}=13 \mathrm{TeV}$ high- $\mu$ dataset over the 2018 data-taking year and (b) the $\sqrt{s}=5 \mathrm{TeV}$ low- $\mu$ dataset in 2017. The stability uncertainties in these two cases are $0.8 \%$ and $0.7 \%$, respectively.

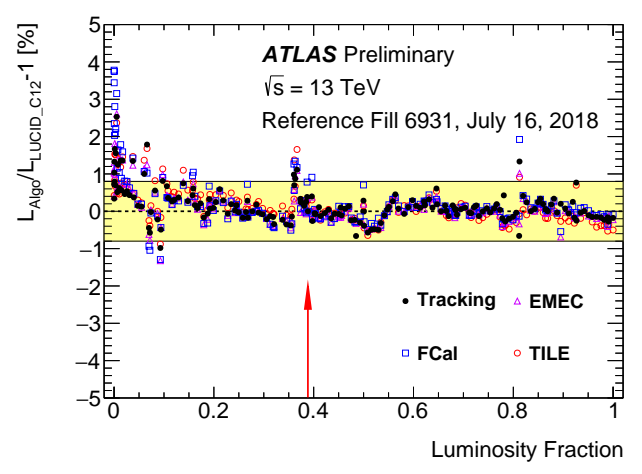

(a)

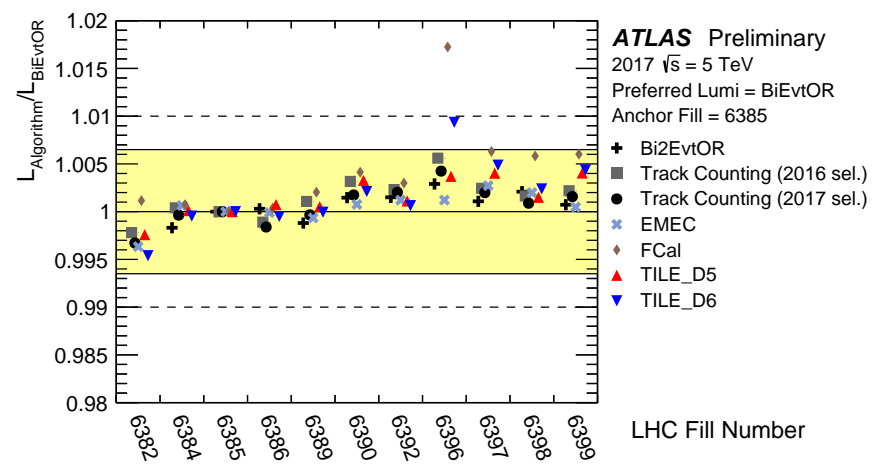

(b)

Figure 3: Long-term stability: Fractional differences in run-integrated luminosity between LUCID and measurements from other detector subsystems for (a) the $\sqrt{s}=13 \mathrm{TeV}$ high- $\mu$ dataset in 2018 [4] and (b) the $\sqrt{s}=5 \mathrm{TeV}$ low $-\mu$ dataset in 2017 [5].

\section{Uncertainties, inter-year correlations and results}

ATLAS physics analyses typically treat datasets with common centre-of-mass energy and running conditions from different years as a single combined dataset and require a single luminosity uncertainty for this dataset. If the total integrated luminosity $\mathcal{L}_{\text {tot }}$ is given by $\mathcal{L}_{\text {tot }}=\sum_{i} \mathcal{L}_{i}$ where $\mathcal{L}_{i}$ is the integrated luminosity of dataset $i$, then its absolute uncertainty $\sigma_{\mathcal{L}_{\text {tot }}}$ is given by $\left(\sigma_{\mathcal{L}_{\text {tot }}}\right)^{2}=$ $\mathbf{G V}_{\mathbf{L}} \tilde{\mathbf{G}}$, where $\mathbf{V}_{\mathbf{L}}$ is the covariance matrix encoding the absolute luminosity uncertainties for the different years and their correlations, and $\mathbf{G}$ is a unit vector.

Using this formulation, a preliminary luminosity uncertainty of $\delta \mathcal{L} / \mathcal{L}=1.7 \%$ was obtained for the Run-2 $\sqrt{s}=13 \mathrm{TeV}$ high- $\mu$ pp dataset [4], with $\delta \mathcal{L} / \mathcal{L}=1.5 \%$ for the $\sqrt{s}=13 \mathrm{TeV}$ low- $\mu$ $p p$ dataset, and $\delta \mathcal{L} / \mathcal{L}=1.5 \%$ for the $\sqrt{s}=5 \mathrm{TeV}$ low- $\mu p p$ dataset [5].

\section{References}

[1] ATLAS Collaboration, JINST 3 (2008) 003.

[2] S. van der Meer, CERN-ISR-PO-68-31, 1968.

[3] P. Grafström and W. Kozanecki, Prog. Part. Nucl. Phys. 81 (2016) 97.

[4] ATLAS Collaboration, ATLAS-CONF-2019-021, June 2019.

[5] ATLAS Collaboration, ATLAS-CONF-2020-023, July 2020.

[6] G. Avoni et al., JINST 13 (2018) P07017. 